\title{
Accrue Discovery Regulation for Autonomous Robotic Control of Marine Cybernetics and Automation
}

\author{
P. S. Jagadeesh Kumar \\ Department of CSE \\ Marathwada Institute of Technology \\ Aurangabad, Maharashtra, India
}

\begin{abstract}
This article expresses the devise of the "Accrue Discovery" regulation for the robotic control of an inundated marine cybernetics and automation. A considerable confederation of momentous relevance can be controlled by permitting such a robot to pursue a plummet or analogous poignant object. The robot exercises a matching set of intellect expertise, chiefly supported by computer vision, allowing it to pilot in flurry and petty marine surroundings. Accrue Discovery (AD) regulation depicted in this article, tolerates the robot to trail and follow a specified object at the bottom of the sea water. The automated marine control is wrapped up of two discrete elements namely; a hunter and a feedback controller. The robotic $\mathrm{AD}$ regulation by means of trifling adoptions can be tattered while the robot might stride and accrue on the earth surface furthermore.
\end{abstract}

\section{General Terms}

Accrue Discovery Regulation, Autonomous Robotic Control, Computer Vision, Intellect Expertise.

\section{Keywords}

Oceanography, Marine Cybernetics and Automation

\section{INTRODUCTION}

With modern efforts, a robot that employs piling motion to swim and to pilot underwater depending on visualization as its principal sagacity has been urbanized in this appraisal. Although sonar is the prime sensor employed by underwater medium, apparition is tremendously effectual in underwater, though its exploitation is sophisticated by numerous aspects. Unequivocally the deployment of visual response to achieve easy routing responsibilities in an underwater ambiance was explored. This is accomplished by possessions of a visual feedback method to acclimatize the swimming pace of this underwater four paddled robot as it tracks a synthetic object conceded by a diver [1]. A mammoth group of considerable appliance can be leveraged by consenting to a robot in order to pursue a diver or analogous poignant object. Performing this underwater is complicated by the erratic lighting and visibility of the shallow sea water. Moreover, the surging motion of this robotic vehicle and the exogenously obsessed motion of the robot in the open water moreover obscure the progression [2].

In this article, the structural design and loom towards robotic control is portrayed. Underwater robotics emerges to be an application vicinity of hurriedly rising connotation, rampant with dispute of both logical and practical impact. Whilst computer vision has developed extremely in the prior decades, the idiosyncrasy of underwater visibility has been basically ignored, necessarily owing to the mammoth logistic and hardnosed transparency in groping them [3]. Without a doubt, visualization can be precious for sensing underwater and perhaps especially crucial sensing deep ocean water than on ground. Effortless study of harbor lay bares the ubiquity of vision and further ocular sensors, in the marine automation and thus proposes its impending efficacy. In this meticulous employment, consequently a diver as it whirls whichever alongside the ground or under sea water. In such incident, a trailing instrument that inflicts a very narrow cognitive load functioning in spite of discrepancy in lighting and impervious in close proximity to wave accomplishment and controls over a temperate range of remoteness [4]. In this paper, vision has the benefit of inert sensing medium and it is thus equally noninvasive and power proficient. Together both are vital concern in a variety of functions varying from ecological assess to precautionary surveillance. Substitute sensing medium like sonar too endure numerous deficit which craft them intricate to use for trailing poignant targets at near location in potentially tumultuous underwater.

\section{OVERVIEW}

The "Accrue Discovery" regulation and the robot control systems are deliberated as a marine swimming robot that is proficient enough to function both on ground as well as underwater. A hysterical successor of the hexapod robot, AD Regulator was fabricated with underwater request in mind, one of which was scrutinizing the marine life. The robot has a water-resistant aluminum safeguard within, in which the electronic equipments and sensors are domiciled as in Fig 1.

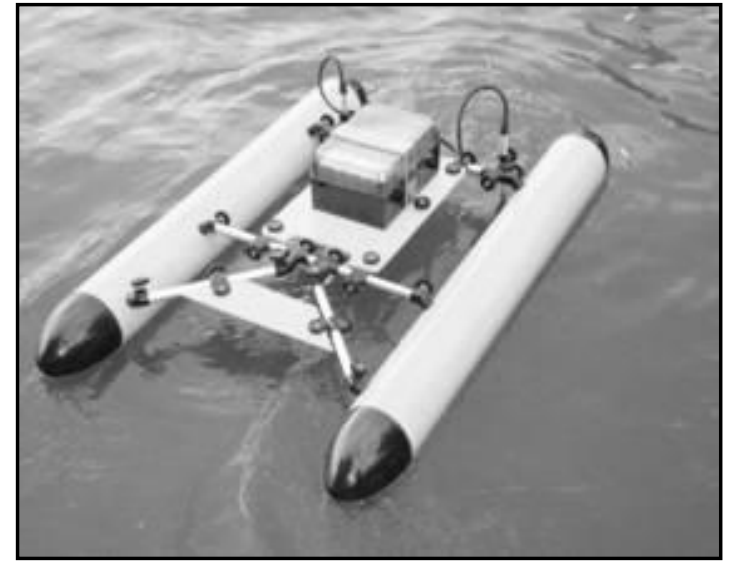

Fig 1: Robot with Accrue Discovery Regulation built up without the paddles at the sea shore

There are five cameras escalated on the robot; two at the fascia and two at the rear and one at the pinnacle. One of the cameras in the fascia affords digital production and has been 
utilized for the regulation mission. The robot can whirl, saunter, uphold position and creep at the base of the sea by means of the four flippers or paddles. With these four paddles the robot can easily control the effective underwater as well as ground movement. These paddles also perform as shell for underwater managing. At the front of the robot is a squat illumination stipulation.

\section{UTLITY OF APPLICATIONS}

The humdrum behavior of underwater travesty troubles the individual in conditions of logistics, outlay, competence and security. By itself, underwater backgrounds signify a substantial vicinity in which robotics can make an expected payment. A variety of appliances can be branded for which effortless scrutiny even in moderately petty water can attest functional. These purposes encompass underwater hunt and salvage, coral vigor supervising, scrutinizing the underwater founding and a lot more [5]. Altogether, in any ecological assessment system, the illustration capacities of a marine bionetwork must be used in periodical. Though instinctively opting for the area of significance is outside the compass of existing expertise, formerly if an ecologist recognizes the area of significance, it can be well understood that a robot may be competent of accruing ancillary information or even autonomously performing assessment expeditions. It is in this milieu that the proposed exertion is outlined [6].

Latest underwater vehicles and sensors in the course of water communication propensity will facilitate innovative progresses in oceanographic inspection, surveillance, and scrutinizing. Groups of preset mobile platforms, as well as submerged automatic control vehicles, autonomous robotic surface vehicles, and fastened gadgets, and gliders will offer relentless spatial exposure, including decisive message and their steering ability. Protectorate and satellite connectivity to the shoreline for surface vehicles might provide human machinist the capacity to explore information and reprogram the vehicles as obligatory. Such an advance may possibly, engage in an amusement of a noteworthy role in durable environmental supervising of underwater oil and gas field predominantly where predictable clout and communications is not easily reached, such as in the investigation phase or following the blackout of a field. The potency of this loom will be pedestal on distinguishing the balancing outlook of dissimilar communication techniques and policies and on merging them in innovative traditions to deal with essential dilemmas [7].

\section{ACCRUE DISCOVERY REGULATION}

In general, "Accrue Discovery" refers to the mission of accumulating, regulating and controlling the pretense of the robot or a control escalated on the robot by means of visual data. In general a closed-loop structure is dilapidated with recent camera specification in diligence, but there have not been comparatively many functions of vehicle control with a permanently laid camera on the robot for car navigation and airplane landing, amongst a few. Even though a number of dissimilar advances to robotic control of visual data subsist, $\mathrm{AD}$ regulation primarily functions by trailing an entity or set of poignant objects in succeeding image frames by accruing, regulating and controlling the robot motors by means of the data offered by the trailer. Performing image based AD regulation; the trailing data is described openly in provisos of image facet stricture, as a replacement of using task space hormones. This can be attained by stirring the robot to an objective location and the matching image is exercised to calculate the set of preferred image facet limits. For implementing image predestined $\mathrm{AD}$ regulation, it is not obliged to figure out the pretense of the aspiration, and it is usually customary that $\mathrm{AD}$ regulation in the image space is further vigorous with veneration to calibration ambiguity than location based. For an entrenched arrangement like $\mathrm{AD}$ regulation, plunging this further overhead betters the general system recital. Autonomous and semi-autonomous robotic vehicles operational with negligible human regulation are currently used in business offshore maneuvers, for security grounds, for classically conducting assessments far from expensive and perceptive infrastructure. Remotely operated vehicle (ROV) procedure, on the contrary, usually happen close to intricate communications, but merely underneath vigilant regulation by expert human pilots. Researchers are practicing a combined research to demonstrate the knack of Autonomous Underwater Vehicles' (AUVs) to confine and conduct in an atmosphere that consist of complex synthetic composition. Such robots will come together with their past experience in navigation and regulation of AUVs in craggy natural territory with their pervasiveness in the calculation technique for robot consignation and navigation in artificial background. Research will start with essential exertion in a test tank fixed with suitable configuration and obstruction, then continue to examining about the waterfront and lastly to a real offshore.

Measurements of disparity or incongruity in Earth's gravitational field endow with precious data concerning the constitution of the seafloor which can assist categorizing thriftily expensive mineral dumps and petroleum coffers. Such dimensions are regularly attained through satellites, but these techniques cannot discover rapid gravity features since the scale of the irregularity reduces as the remoteness amid the seafloor and the sensor augments. Exemplifying this inconsistency entails gravity measurements from inundated platforms proficient of commissioning next to the seafloor. Piloted and tenuously controlled vehicles are too expensive for such dimensions; AUVs offer a cost effectual substitute. Researchers and business purveyors are raising a new-fangled set of gravimeters that are reduced in size and low power obligatory traits for AUVs implementation and firmly attached to the navigation sensors on the robot. Instant to this exertion, research is conceded into enhanced routing and dexterous control techniques that will progress the precision and spatial decree of AUVs gravity magnitude. Control effort spotlights in merging presently accessible bathymetric atlas and instantaneous sensor data to curtail both the aloofness from the seafloor and the vehicle hastening. Navigational advancement highlights on new techniques for approximating vehicle speeding to amplify the capacity to accurate vehicle movement and thus advance the excellence of underway gravimeter dimensions.

\section{AUTONOMOUS ROBOTIC CONTROL}

The dichotomy outside edged by effusively autonomous underwater robotics and robots proscribed straightforwardly by individuals is quickly on its last legs. Remotely operated vehicles, which are conventionally prohibited by a diver, are fitting to be more automatic. Undeterred vehicles at present embrace more and more consistent and competent auditory infrastructure acquaintances that facilitate connoisseurs on the 
facade to utilize information from the vehicle to preplan tasks accordingly. Subsequent to a current manifestation of highspeed, short-distance, underwater optical messages to manage an undeterred ROV in concurrent, scientists are presently betrothed in research to decide how eminently to coalesce automation with human acquaintance and ability to perk up vehicle competence. The idea of co-robotics just materialized to portray robots that they labor alongside with human. Researchers are expanding this to incorporate robots functioning with groups of human on nearby sustain craft and connected via the Internet. This attempt will merge idea from software engineering, human features, and networking techniques to discover the most excellent traditions to put into practice the co-robotic control method.

Robotic systems give surety to unlock the ocean to humans in novel methods. Modern succession in robotics are civilizing effectively by plummeting capital, and plunging the jeopardy of marine procedures. Robots also permit science, diligence and the martial to deal with the predicaments by new approaches, habitually fabricating resolutions that cannot be attained through conventional means. The goal of the oceanography is to join forces with diligence, academic world, and key government society to improve the way human and robots labor together in the marine atmosphere. Many marketable purveyors have begun joint research of Marine Robotics with scientists, production personnel, belligerent users and developers in producing the next generation of independent and tenuously activated ocean systems. Remotely operated vehicle (ROV) systems have conventionally used deters to relay power and control signals amongst vehicles whereas by the same instance broadcasting sensor information and first-class video to the ground. Deters also play momentous fetters on vehicle functions. Cranes and cables are essential to sustain the vehicles, for instance, they are pretty huge and can uphold deepwater processes to big vessels with vibrant introduction competency. Scientists have residential a multi-operational, optical transmitting system that get rid of a lot of these responsibilities while still providing standard, less suppressed video that pilots necessitate for manipulator task. The composition's optical modem meets the expenditure of associations up to $20 \mathrm{Mbit} / \mathrm{s}$ operational by 150 meters of water. With suitable video compression, the optical modem can prop up instant video. The technique that was recently positioned consist of battery power-driven automobile that recognized the latent to run the vehicle, to seize taster, and execute vocation with no deter. The trailer is erected on the principle of area of significance machinist. In this paper, a color bead trailer which sections out a meticulous province pedestal on the color constraints that are specified as input to the trailer is established.

A proportional-derivative (PD) controller as exposed in Fig 2 is upright. Controller gets the fault messages from the trailer and produce terrain and way pedals for the walk controller. In turn to preserve the goal in the middle of the camera's vision, two feedback loops are indispensable. The complete implement of a fittingly refrained PID controller with further modification of the walk controller would assist trim down and conceivably eradicate those fluctuations. The frame rate worned by the visual preview is 1 frame/second, consistent with the low-pass control loop consented by the swinging saunter. Enhanced underwater communications is facilitating humans and robots to work hard jointly, further efficiently to determine complicated troubles that have not conventionally been acquiescent to automation.

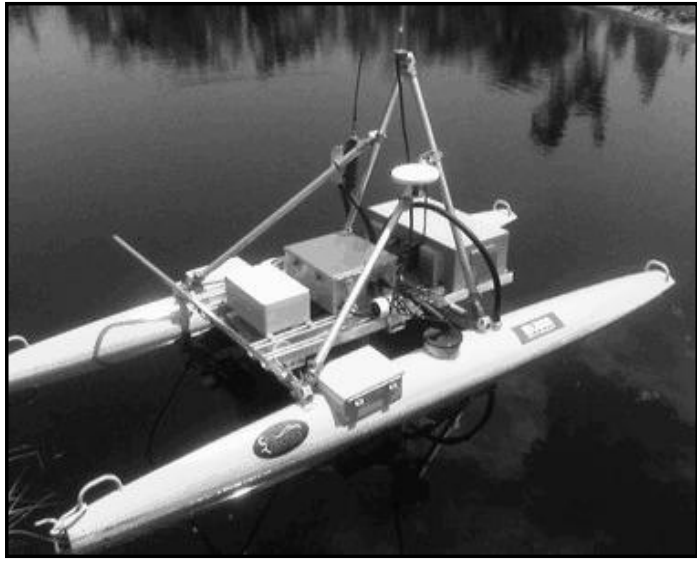

Fig 2: Robot with PD Controller and AD Regulator built up without the paddles at the sea shore

Underwater robots move away from their earthly, in-flight, and space complement in that when they are inundated, they can never take advantage of radio frequencies as seawater alleviates electromagnetic radiations in surfeit of squat hodgepodge. Scientists recently industrialized and patented a free-space, underwater optical structure using luminosity to broadcast information all the way through water. This scheme presents unparalleled bandwidth (1 to $20 \mathrm{Mbit} / \mathrm{s}$ ) which varies from 500 meters. Joint with further underwater acoustic expertise, the method can also offer more reticent bandwidths. In a topical research, researchers engaged a mishmash of optical and acoustic modems to conduct sampling and exploitation maneuvers by means of an undeterred ROV. These tools ease an innovative cohort of AUVs and undeterred ROVs with the intention of communicating with one another and with controllers on the peripheral devoid of a corporeal association and are able to offload huge information files from sovereign apparatus. These vehicles exploit customary engineering resources, in fastidious titanium for potency and syntactic foam for resilience. To deposit up the crossbreed AUVs/ROV engineers resolute that more superior structural resources would be desired to attain the complete depth of the ocean, though at the same instance gathering sensible restraints on mass and heaviness. A narrative legion of one atmosphere pressure covering to hold the crossbreed tools, cameras, and batteries were hand-me-down. Exceptionally optimized ceramic orbs were preferred to propose standard vehicle optimism. Ceramics resources have a great deal of strength-to-weight proportion standards to unadventurously profound submergence structural equipments and facilitate them to demonstrate at the most tremendous deepness. For the subsequent relations of ocean journeying coordination, ceramics guarantee to decrease the heaviness vital for chief vehicle configuration and malleability, most important to a considerable augment in vehicle consignment, fortitude and competence.

\section{MARINE CYBERNETICS AND AUTOMATION}

Marine cybernetics and automation is defined to be the knowledge concerning procedures and methods for analysis, monitoring and organize of marine coordination as in Fig 3. 


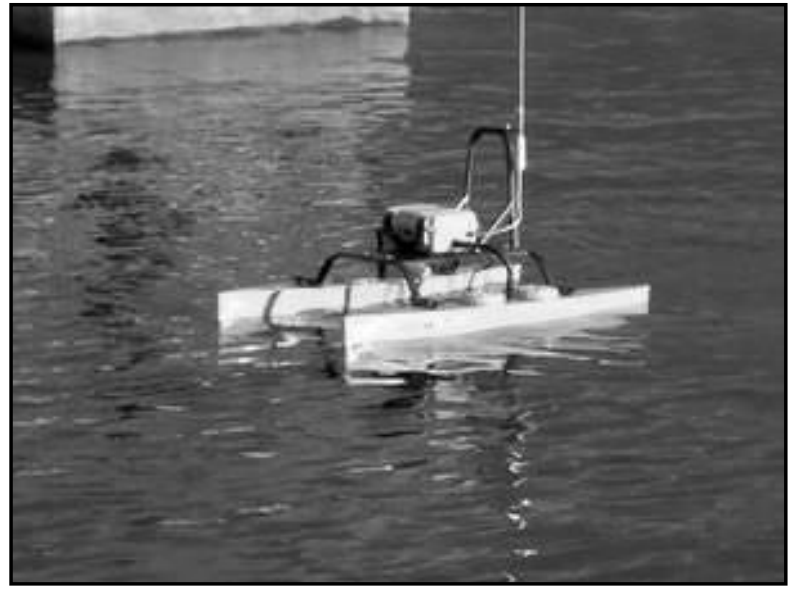

Fig 3: AD Regulated and Robotic Controller at the sea shore built up with Marine cybernetics and Automation

Nowadays offshore engineering is extremely reliant on control systems that make certain appropriate functions of vessels and assembles. It is accordingly significant to check if those schemes work sufficiently well. If a ship's Dynamic Positioning System stops working, it could effect in thrashing of location, which consecutively could guide to clash. If bradawl base software has concealed faults, it will frequently guide to non-prolific-moment: bradawl cannot continue in anticipation of the software trouble is resolved. By means of the CyberSea knowledge, one is capable to execute a Hardware-In-the-Loop (HIL) simulation to check virtually any control system for blunders and heftiness. Marine Cybernetics utilizes our CyberSea expertise to check and substantiate control system software. HIL ordeal is a very well demonstrated test tactic from space, automotive, avionics, robotics, power electronics, and nuclear industries. It assist methodical taxing of control system blueprint idea, utility, recital, and stoppage management capability, both in standard and off-design servicing provisions. This testing is executed in an implicit test bed where there is no jeopardy to human, ship, or paraphernalia. Testing is consummate by involving the control system to a craft precise simulator or offshore mechanism. The simulator retorts to control signals in a sensible manner, and affords pragmatic and reliable dimensions. The control system cannot mind any disparity between the existent world and the implicit world in the simulator. The control system is further experienced in pragmatic working circumstances and breakdown situation pedestal on a craft precise test agenda that insists for the definite tackle, devise and outfitted beliefs of the ship. In the nautical and offshore diligence that class civilization and corporation have a protracted and thriving ritual as provider for third party taxing and corroboration. Autonomous is a mean attribute to facilitate the indispensable intensity of substantiation security and assignment decisive for control systems. Knowledge has revealed that a huge figure of controls system inaccuracy and limitation lies in the boundary amid dissimilar control systems.

Official recognition of the border amid individual structure and their communal utility is a consequence of high significance to accomplish the essential echelons of dependability, protection and recital. Whenever the critical systems are distributed by diverse merchants, only a third party can execute an impartial inspection of the incorporated utility exclusive of sensational business data involving the concerned festivities. Third party taxing does not reinstate interior test behavior by the retailer or vice versa, even if together the third party and internal examination are done by means of HIL testing; both are imperative tricks for attaining high feature software and gathering essential principles for security significant control systems. Devoid of third party investigation, the system software will not have been practiced by someone apart from the system purveyor at deliverance of the vessel. Control system software is a necessary and incorporated ingredient of all contemporary vessels. From small entrenched control systems on board and sensors to the vastly multifaceted dynamic positioning computer systems, software constitutes an intrinsic fraction of a huge number of security and assignment significant automation systems. Further paradigms are drilling control systems, Power management systems, BOP control systems and crane control systems. Illustration of occurrence and reoccurrence might be grounded by the stoppage in these methods causing slaughter of location owing to drive off or drift off, absolute or biased block out, injure to the health, and perhaps fire/explosion as a derivative of the failure. Simulator based testing, is a well-organized and efficient device to represent the ability and sturdiness of the control system software. In newly constructed projects the effort is to synchronize and organize liberation from several control system purveyors.

\section{CONCLUSION}

The Accrue Discovery (AD) regulation for autonomous robotic control of marine cybernetics and automation is intrinsically trouble-free and facilitate to accomplish some measure of self-determination in navigating underwater. The theory of $\mathrm{AD}$ regulation is pedestal on simple color trailing together a control loop with low-pass chattels refrained to purge the normal furrows caused by the robots plummeting pace. The sea tryouts of the proposed system proved to be exceedingly thriving, and explores exhilarating new trends in marine robotics. It shows that new supple learning based methods for target attainment and trailing would consent the scheme to function more strongly with no staid occurrence of trailing crashes. Lighting averts the target from attained; one might anticipate this to transpire in the dearth of on-line automation. Significantly, it materializes that the trailing system can be "hoodwinked" by troubling substances whose tinge goes with that of concern target. Despite the fact that by means of accompanying shape-based signals would be a natural enhancement to the hunter, the calculation slither, predominantly in the robot's diminutive form factor makes it challenging for the future work. A foretaste pedestal on color histograms or allied color normalization also appears demanding, but in underprivileged visibility situations the variety of existing shades broadcast through the underwater is enormously narrow. A predominantly pretty alternative is the utilization of a trailer that unequivocally sculpts the faction of the objective. Hitherto, the robotic AD regulation by means of trivial espousals can be dilapidated whilst the robot might tramp and accumulate on earth's facade as well.

\section{REFERENCES}

[1] James Paulos, Nick Eckenstein, Tarik Tosun, Jungwon Seo, Jay Davey, Jonathan Greco, Vijay Kumar, and Mark Yim,"Automated Self-Assembly of Large Maritime Structures by a Team of Robotic Boats," IEEE Transactions on Automation Science and Engineering, 
Vol.3,

2015, pp.

958-968, doi:10.1109/TASE.2015.2416678.

[2] Peter Simon Sapaty, "Military Robotics: Latest Trends and Spatial Grasp Solutions," International Journal of Advanced Research in Artificial Intelligenc, Vol. 4, No.4, 2015, pp.9-18, doi: 10.14569/IJARAI.2015.040402.

[3] Jorge M. Soares, A. Pedro Aguiar, Antonio M. Pascoal, and Alcherio Martinoli,"Design and Implementation of a Range-Based Formation Controller for Marine Robots," ROBOT2013: First Iberian Robotics Conference, Advances in Intelligent Systems and Computing, Vol. 252, 2014, pp. 55-67, doi:10.1007/978-3-319-03413-3_5.

[4] Emaad Mohamed H. Zahugi, Mohamed M. Shanta and T.V.Prasad, "Degign of Multi-Robot Ssystem for
Cleaning up Marine Oil Spill," International Journal of Advanced Information Technology, Vol. 2, No.4, 2012, pp.33-43, doi:10.5121/ijait.2012.2404.

[5] Yogesh Girdhar, Anqi Xu, Bir Bikram Dey, Malika Meghjani, Florian Shkurti, Ioannis Rekleitis, and Gregory Dudek,"MARE: Marine Autonomous Robotic Explorer," IEEE/RSJ International Conference on Intelligent Robots and Systems, September 25-30, 2011, pp. 5048-5053, doi:10.1109/IROS.2011.6094914.

[6] Neil Munro, Frank L.Lewis, "Robot Manipulator Control Theory and Practice," Second Edition," 2004, Marcel Dekker, New York, Basel.

[7] J.Aranda, M.A.Armada, J.M.de Cruz, "Automation for the Maritime Industries," 2004, Producción Grafica Multimedia, Madrid, Spain. 